\section{Qualidade de vida de pacientes idosos vivendo com HIV/AIDS}

\author{
Quality of life in elderly patients living with \\ HIV/AIDS
}

\author{
La calidad de vida de los pacientes de edad \\ avanzada que viven con el VIH/SIDA
}

\author{
1 Universidade Federal de \\ São Paulo, São Paulo, Brasil. \\ Correspondência \\ M. F. P. Okuno \\ Universidade Federal de São \\ Paulo. \\ Rua Napoleão de Barros 754 \\ São Paulo, SP 04024-002, \\ Brasil. \\ mf.pinto@unifesp.br
}

\section{Abstract}

This study's objective was to evaluate quality of life in older adults living with HIVIAIDS and the associations with socio-demographic, economic, and clinical characteristics, using a cross-sectional design. Data were collected on demographics, disease history, and economic status according to the Brazilian Economic Classification Criterion and Targeted Quality of Life Instrument (HAT-QoL). A total of 201 elderly participated, with the following characteristics: males (63.7\%), single or divorced (51.3\%), economic classes C, D, or E (61.7\%), and low schooling (53.7\%). Nearly two-thirds (130, or 64.7\%) had been infected by the sexual route. The following were significantly correlated with two or up to seven domains in the HAT-QoL: age, individual and family income, and time since diagnosis. Quality of life in elderly living with HIVI AIDS showed the lowest scores in the domains related to concern over disclosure of their diagnosis, sexual activity, and financial concerns. Age, income, and time since diagnosis were the variables that most accounted for altered quality of life.

Aged; HIV; Quality of Life
Meiry Fernanda Pinto Okuno 1

Alexandre Cavallieri Gomes 1

Letícia Meazzini 1

Gerson Scherrer Júnior 1

Domingos Belasco Junior 1

Angélica Gonçalves Silva Belasco 1

\section{Resumo}

O objetivo foi avaliar a qualidade de vida de idosos com HIVIAIDS e correlacioná-la às características sociodemográficas, econômicas e clínicas. Estudo transversal analítico que avaliou idosos que vivem com HIVIAIDS. Coletadas informações sociodemográficas, mórbidas e situação econômica de acordo com o Critério de Classificação Econômica Brasil, $e$ Targeted Quality of Life Instrument (HATQoL) que avalia qualidade de vida. Participaram 201 idosos, do sexo masculino (63,7\%), solteiros ou divorciados (51,3\%), classe econômica C, D ou E (61,7\%) e baixa escolaridade (53,7\%). A forma de contágio prevalente foi a sexual 130 (64,7\%). Apresentaram correlação significativa com dois ou até sete domínios do HAT-QoL: idade, renda individual e familiar, e tempo de diagnóstico. A qualidade de vida dos idosos que vivem com HIVI AIDS apresentou menores escores nos domínios preocupação com o sigilo, atividade sexual e preocupação financeira. Idade, renda e tempo de diagnóstico foram as variáveis que mais responderam pela alteração da qualidade de vida.

Idoso; HIV; Qualidade de Vida 


\section{Introdução}

Trinta anos após a primeira descrição da síndrome da imunodeficiência adquirida (AIDS), casos da doença são diagnosticados em todas as partes do mundo. Mais de 34 milhões de pessoas estão infectadas e a maioria delas vive em países com baixa e média renda econômica 1 .

A prevenção e controle do vírus da imunodeficiência humana (HIV) têm sido um grande desafio para a área da saúde nas últimas três décadas. A pandemia gerada pelo HIV é de natureza complexa, pois não há cura para a doença, a propagação é persistente, atinge especialmente populações mais pobres e indivíduos que se expõem a comportamento de risco, neste estudo considerado como atividades praticadas por indivíduos, com frequência e intensidade tal, que conduzam ao aumento de risco de adquirir doença ou acidentar-se, e neste caso práticas que levam o indivíduo à menor ou maior grau de exposição ao HIV, como: sexo desprotegido, com profissional do sexo, com parceiro do mesmo sexo, uso de drogas ilícitas e transfusão sanguínea 2 .

O comportamento sexual de risco como o sexo desprotegido e o uso de drogas ilícitas, muitas vezes atribuídos aos adolescentes e adultos jovens, também pode refletir o comportamento dos adultos mais velhos e idosos. Dessa forma, os idosos devem ser incluídos nas campanhas de prevenção da AIDS ${ }^{3}$. Com o advento e acesso às novas drogas antirretrovirais, o uso correto das mesmas tem possibilitado melhor controle da doença e redução das morbidades e da mortalidade principalmente secundária a infecções oportunistas 4 .

A AIDS é considerada uma doença crônica que pode ser prevenida por meio de abordagens e medidas sistemáticas e efetivas de curto, médio e longo prazos 4 . O tratamento permite sobrevida prolongada em muitos casos, entretanto, a acessibilidade e a aceitabilidade ainda permanecem como obstáculos contínuos para o tratamento adequado em diversas partes do mundo 5 .

A infecção pelo HIV atinge indivíduos em qualquer idade, porém, entre adolescentes e idosos a incidência vem aumentando nos últimos anos. Esse perfil difere do início da doença, quando eram os grupos menos afetados 3 .

Viver com HIV e manifestar a doença continuam sendo condições estressantes e que exigem muito do doente nos aspectos: psicológico, físico, social, ambiental, entre outros 6 . Existem evidências de que o estresse pode acelerar a progressão do HIV/AIDS, aumentando a replicação viral e suprimindo a resposta do sistema imunológico ${ }^{7}$. Além disso, os soropositivos também podem enfrentar problemas como depressão, isolamento, estigmatização e discriminação, que podem levar a eventos traumáticos de vida e causar impacto negativo e progressivo no curso da doença 8 .

O estigma é um processo dinâmico de desvalorização que desacretida fortemente um indivíduo perante os outros, produzindo e reproduzindo relações de poder assimétricas. As característas relacionadas ao estigma são arbitrárias, assim como, certas condições de saúde, sexo, raça, comportamento sexual, entre outras. Discriminação é a manifestação da estigmatização e consiste em todas as formas de exclusão ou restrição, seja por ação ou por omissão, com base em característica pessoal inerente ou perceptível relacionada a determinado grupo. Estigmatização e discriminação interferem nas diferenças individuais e nas desigualdades sociais e estruturais 9 .

As características descritas no início da epidemia de HIV/AIDS que definia a doença como contagiosa, incurável, mortal e ligada a homossexuais, vinculou-a aos estigmas e preconceitos advindos da posição social, da forma de contaminação e do comportamento sexual que ainda continuam arraigados na sociedade ${ }^{9}$.

No início da epidemia de AIDS, não era esperado que pessoas infectadas, ainda jovens, vivessem até a idade avançada, e os mais idosos, em geral, não eram considerados grupo de risco para contrair a infecção. Entretanto, o advento dos medicamentos que melhoram o desempenho sexual e possibilitam o estabelecimento de novas e múltiplas parcerias sexuais, e a disponibilidade da terapia antiretroviral altamente ativa (HAART) anteriormente não existente, têm favorecido a infecção pelo HIV de pessoas com mais idade, porém, com possibilidade de sobrevida maior quando tratadas adequadamente 10 .

A HAART permitiu maior controle da doença que de progressiva e fatal passou a ser considerada tratável e crônica. Porém, o controle da replicação do HIV, por meio da terapia medicamentosa eficaz, traz consigo inúmeros efeitos adversos capazes de comprometer significativamente a qualidade de vida e, consequentemente, a adesão ao tratamento 11 .

Qualidade de vida é um conceito multidimensional que inclui questões econômicas, estilo de vida, condições de saúde, habitação, satisfação pessoal e do ambiente social, entre outras. É conceituada de acordo com um sistema de valores, normas ou perspectivas que variam de pessoa para pessoa, de grupo para grupo e de lugar para lugar, de modo que a estrutura conceitual da qualidade de vida integra, entre outras coisas, o sentimento de bem-estar que representa a soma de sentimentos indivíduais 12 . 
A pessoa que vive com HIV/AIDS e enfrenta dificuldades para o acesso e má qualidade no tratamento pode manifestar problemas relacionados à adesão, o que aumenta potencialmente o risco de transmissão da doença e redução da qualidade de vida 13 .

A qualidade de vida de indivíduos com HIV/ AIDS tem sido associada às características sociodemográficas, econômicas e clínicas como: idade, gênero, etnia, nível educacional, renda, emprego e tipo de acesso aos cuidados de saúde, além da própria condição clínica do paciente 14 .

Este estudo teve por objetivo avaliar a qualidade de vida dos idosos com HIV/AIDS e correlacioná-la às variáveis sociodemográficas, econômicas e clínicas por eles apresentadas.

\section{Métodos}

Estudo epidemiológico, transversal e analítico aprovado pelo Comitê de Ética em Pesquisa da Universidade Federal de São Paulo (CEP Unifesp 0182/11), que investigou 201 idosos com diagnóstico de HIV/AIDS atendidos na unidade ambulatorial coordenada pela Disciplina de Doenças Infecciosas e Parasitárias da Unifesp. Todos os participantes do trabalho assinaram o termo de consentimento livre e esclarecido, antes do início da entrevista, e tiveram as suas identidades preservadas.

Foram considerados para o estudo pacientes com sorologia positiva para o HIV, de ambos os sexos, com idade mínima de 50 anos, classificados como idosos quando portadores desta doença, segundo a classificação do Centers for Disease and Control and Prevention dos Estados Unidos (CDC) e do Programa Conjunto das Nações Unidas sobre HIV/AIDS (UNAIDS) 15,16, que aceitaram participar do estudo e assinaram o termo de consentimento livre e esclarecido. Perguntas em relação à orientação sexual não foram feitas aos pacientes desta pesquisa. As entrevistas com os pacientes foram realizadas nos dias agendados para a coleta de exames ou consulta médica, em ambiente privado, no período de maio de 2011 a março de 2012, e com base na aplicação de questionários. O primeiro para medir as variáveis de caracterização sociodemográfica, mórbida e linfócitos CD4; o segundo para caracterizar a situação econômica, Critério de Classificação Econômica Brasil - CCEB (Associação Brasileira de Empresas de Pesquisa. http://www.abep.org/novo/ Content.aspx?ContentID=302, acessado em 15/ Out/2013); e o terceiro, específico, para avaliar a qualidade de vida de pessoas com HIV/AIDS Targeted Quality of Life Instrument (HAT-QoL) 17.
O CCEB, elaborado pela Associação Brasileira de Empresas de Pesquisa, foi obtido por meio da conversão da soma de pontos referentes ao grau de instrução do paciente pesquisado e a quantidade de bens de consumo existentes em cada casa, em uma das classes econômicas (A a E).

O HAT-QoL considera informações referentes às últimas quatro semanas e é constituído por 34 perguntas, que compõem as seguintes dimensões: função geral, satisfação com a vida, preocupações com a saúde, preocupações financeiras, preocupações com a medicação, aceitação do HIV, preocupações com o sigilo, confiança no médico e atividade sexual. Para cada questão há cinco opções de respostas: todo o tempo, a maior parte do tempo, parte do tempo, pouco tempo, nunca. A soma das respostas atribuídas aos itens de cada dimensão da escala permite obter o escore total de cada dimensão que varia de 0 (pior estado) a 100 (melhor estado).

Foi utilizada a análise descritiva dos dados para a caracterização sociodemográfica, econômica, clínico-epidemiológica, comorbidades e atividade física realizada pelos pacientes. Para as variáveis contínuas calcularam-se a média, desvio-padrão, mediana, mínimo e máximo, e para as variáveis categóricas calcularam-se a frequência e o percentual. Para verificar quais as variáveis contínuas se relacionavam com cada domínio do HAT-QoL utilizou-se a correlação de Spearman, considerando-se um nível de 5\% de significância (valor de $\mathrm{p} \leq 0,05$ ).

\section{Resultados}

Neste estudo, houve predomínio de pacientes do sexo masculino, com cor da pele branca, solteiros ou divorciados, aposentados ou pensionistas e das classes econômicas mais baixas (Tabela 1).

Dentre os pesquisados o tempo de diagnóstico da doença variou de seis meses a trinta anos, com média de 12 anos; a via sexual foi referida como a principal forma de infecção pelo HIV, $46,2 \%$ apresentavam $\mathrm{CD} 4 \leq 350 / \mathrm{mm}^{3}$ no mês em que foi feito o diagnóstico da doença. As doenças cardiovasculares aparecem como as comorbidades mais prevalentes e cerca da metade dos entrevistados realizava algum tipo de atividade física de rotina (Tabela 2).

$\mathrm{Na}$ avaliação da qualidade de vida dos idosos que vivem com HIV/AIDS, os domínios do HAT-QoL com menores escores médios foram: preocupação com o sigilo, atividade sexual e preocupação financeira (Tabela 3).

As variáveis idade, tempo de diagnóstico, renda individual e familiar apresentaram correlação significante de fraca magnitude com no mínimo 
Características sociodemográficas e econômicas dos pacientes com HIV/AIDS. Universidade Federal de São Paulo, São Paulo, Brasil, maio de 2011 a março de 2012 ( $N=201)$.

\begin{tabular}{|c|c|}
\hline Características & Mediana (mínimo-máximo)/n (\%) \\
\hline Idade (anos) & $56(50-74)$ \\
\hline \multicolumn{2}{|l|}{ Sexo } \\
\hline Masculino & $128(63,7)$ \\
\hline Feminino & $73(36,3)$ \\
\hline \multicolumn{2}{|l|}{ Cor da pele } \\
\hline Branca & $136(67,7)$ \\
\hline Negra & $27(13,4)$ \\
\hline Parda & $38(18,9)$ \\
\hline \multicolumn{2}{|l|}{ Estado civil } \\
\hline Solteiro/Divorciado & $103(51,3)$ \\
\hline Casado & $65(32,3)$ \\
\hline Viúvo & $33(16,4)$ \\
\hline \multicolumn{2}{|l|}{ Ocupação } \\
\hline Aposentado/Pensionista & $106(52,7)$ \\
\hline Empregado & $76(37,8)$ \\
\hline Desempregado & $10(5,0)$ \\
\hline Dona de casa & $9(4,5)$ \\
\hline \multicolumn{2}{|l|}{ Escolaridade } \\
\hline Não letrado/Ensino Fundamental incompleto & $64(31,8)$ \\
\hline Ensino Fundamental & $44(21,9)$ \\
\hline Ensino Médio & $64(31,8)$ \\
\hline Superior & $29(14,4)$ \\
\hline \multicolumn{2}{|l|}{ Renda mensal (Reais) } \\
\hline Individual & $1.200(0-9.000)$ \\
\hline Familiar & $0(0-16.000)$ \\
\hline Indivíduos dependentes da renda familiar & $2(1-9)$ \\
\hline \multicolumn{2}{|l|}{ Classe econômica } \\
\hline$A+B$ & $77(38,3)$ \\
\hline$C+D+E$ & $124(61,7)$ \\
\hline
\end{tabular}

dois e até sete domínios do HAT-QoL, com exceção dos domínios preocupação com a medicação e confiança no médico. A idade apresentou correlação com as dimensões: preocupação financeira $(\mathrm{r}=0,18)$, preocupação com o sigilo $(\mathrm{r}=-0,16)$ e atividade sexual $(\mathrm{r}=-0,25)$. O tempo médio de conhecimento do diagnóstico mostrou correlação com as dimensões do HAT-QoL: satisfação com a vida $(\mathrm{r}=0,14)$, preocupação com a saúde $(\mathrm{r}=$ $0,20)$, aceitação do HIV $(r=0,14)$ e preocupação com o sigilo $(r=0,14)$. A renda individual mostrou correlação com as dimensões do HAT-QoL: função geral $(r=0,22)$, satisfação com a vida $(r=0,22)$, preocupação com a saúde $(\mathrm{r}=0,26)$, aceitação do HIV $(r=0,18)$, preocupação com o sigilo $(r=-0,19)$ e atividade sexual $(r=0,23)$, e a renda familiar com as dimensões preocupação com a saúde $(r=-0,19)$ e preocupação com o sigilo $(\mathrm{r}=0,15)$ (Tabela 4$)$.

\section{Discussão}

Os idosos deste estudo apresentaram menores escores médios nos domínios do HAT-QoL: preocupação com o sigilo, atividade sexual e preocupação financeira. Esses resultados foram semelhantes aos encontrados por outro trabalho realizado em Porto Alegre (Rio Grande do Sul), com pacientes a partir de 50 anos com HIV em que os mesmos domínios forma os mais comprometidos, preocupação com o sigilo, atividade sexual e preocupação financeira 18 .

A preocupação com o sigilo apresentada pelos idosos pode estar relacionada ao estigma associado à doença, que se refere à desvalorização discriminação e julgamento do modo de vida e comportamento das pessoas que vivem com o vírus 19 . 
Características clínicas, epidemiológicas, comorbidades e atividade física dos idosos que vivem com HIV/AIDS. Universidade Federal de São Paulo, São Paulo, Brasil, maio de 2011 a março de 2012 (N = 201).

\begin{tabular}{lc}
\hline Características & Mediana (mínimo-máximo)/n (\%) \\
\hline Tempo de diagnóstico da infecção (anos) & $12(0,5-30,0)$ \\
Forma de contágio & \\
$\quad$ Não sabe & $48(23,8)$ \\
$\quad$ Via sexual & $130(64,7)$ \\
$\quad$ Via parenteral * & $23(11,5)$ \\
CD4/mm sangue (mês do diagnóstico) [n=197] & \\
$\leq 200$ & $49(24,9)$ \\
$>200$ e $\leq 350$ & $42(21,3)$ \\
$>350$ e $\leq 500$ & $46(23,4)$ \\
$>500$ & $60(30,5)$ \\
CD $4 /$ mm ${ }^{3}$ sangue (mês da entrevista) [n $\left.=197\right]$ & \\
$\leq 200$ & $10(5,1)$ \\
$>200$ e $\leq 350$ & $31(15,7)$ \\
$>350$ e $\leq 500$ & $39(19,8)$ \\
$>500$ & $117(59,4)$ \\
Comorbidades & \\
Nenhuma & $59(29,4)$ \\
Doenças cardiovasculares ** & $69(34,3)$ \\
Retinopatia & $49(24,4)$ \\
Neoplasia & $24(11,9)$ \\
Atividade física *** & \\
Sim & $106(52,7)$ \\
Não & $95(47,3)$ \\
\hline
\end{tabular}

* Formas de contágio parenteral relatadas: drogas injetáveis, perfurocortantes, transfusão sanguínea e tratamento dentário;

** Doenças cardiovasculares: hipertensão arterial, doença vascular periférica e coronariopatia;

*** Atividade física: caminhar, andar de bicicleta, nadar e atividades em academia de ginástica.

Tabela 3

Valores médios dos escores dos domínios do Targeted Quality of Life Instrument (HAT-QoL), de pacientes com HIV/ AIDS. Universidade Federal de São Paulo, São Paulo, Brasil, maio de 2011 a março de 2012 ( $N=201)$.

\begin{tabular}{lc}
\hline Domínios HAT-QoL & Média (desvio-padrão) \\
\hline Função geral & $79,29(20,87)$ \\
Satisfação com a vida & $71,89(24,42)$ \\
Preocupação com a saúde & $83,24(22,90)$ \\
Preocupação financeira & $55,64(37,51)$ \\
Preocupação com a medicação & $88,72(17,30)$ \\
Aceitação do HIV & $77,80(33,57)$ \\
Preocupação com o sigilo & $39,03(27,27)$ \\
Confiança no médico & $72,22(30,71)$ \\
Atividade sexual & $45,96(43,50)$ \\
\hline
\end{tabular}

A atividade sexual pode estar prejudicada nos indivíduos soropositivos para o HIV pela preocupação em transmitir o vírus, falta de confiança e dificuldade em negociar o uso de preservativo com parceiro 18 .

Outra pesquisa demonstrou que a baixa renda influenciou negativamente na avaliação da qualidade de vida dos participantes que recebiam até um salário mínimo mensal e que houve relação entre baixa renda e comprometimentos no estado geral de saúde 20 .

Foram constatadas correlações significantes, porém de fraca magnitude, entre as dimensões do HAT-QoL e as variáveis idade, tempo de diagnóstico, renda individual e renda familiar. Estudo realizado na Região Nordeste do Brasil com pessoas vivendo com HIV a partir de 50 anos demonstrou que as variáveis citadas anteriormente interferiram na qualidade de vida, como apontado nesta pesquisa 20 . 
Correlação entre os domínios do Targeted Quality of Life Instrument (HAT-QoL) e as características sociodemográficas, econômicas e clínicas. Universidade Federal de São Paulo, São Paulo, Brasil, maio de 2011 a março de 2012 (N = 201 ).

\begin{tabular}{|c|c|c|c|c|}
\hline Domínios HAT-QoL & $\begin{array}{l}\text { Idade } \\
\qquad r^{2}\end{array}$ & $\begin{array}{c}\text { Tempo diagnóstico } \\
r^{2}\end{array}$ & $\begin{array}{l}\text { Renda individual } \\
\qquad r^{2}\end{array}$ & $\begin{array}{c}\text { Renda familiar } \\
r^{2}\end{array}$ \\
\hline Função geral & - & - & 0,22 * & - \\
\hline Satisfação com a vida & - & 0,14 ** & 0,22 * & - \\
\hline Preocupação com a saúde & - & 0,20 * & 0,26 * & $-0,19 \star \star$ \\
\hline Preocupação financeira & 0,18 * & - & $0,47 *$ & - \\
\hline Preocupação com a medicação & - & - & - & - \\
\hline Aceitação do HIV & - & 0,14 ** & $0,18 * \star$ & - \\
\hline Preocupação com o sigilo & $-0,16 * \star$ & 0,14 ** & $-0,19$ * & 0,15 * \\
\hline Confiança no médico & - & - & - & - \\
\hline Atividade sexual & $-0,25 *$ & - & 0,23 * & - \\
\hline
\end{tabular}

r2: correlação de Spearman.

* Valor de $\mathrm{p}<0,01$;

** Valor de $p<0,05$

O impacto da infecção pelo HIV pode gerar mudanças em diversos aspectos da vida dos indivíduos. Enfrentar esse problema, associado às dificuldades que a condição sorológica impõe em relação à qualidade de vida, tem sido um dos desafios enfrentados pelas pessoas que vivem com HIV/AIDS. Situação de dificuldade como essa também é enfrentada pelo idoso que vive com uma doença estigmatizada, sem cura e que exige suportes social, familiar e profissional para ser enfrentada e vivida 21 .

Apesar dos tabus existentes sobre a sexualidade do idoso, o aumento de casos de AIDS em pessoas com idades mais avançadas pode estar relacionado à disponibilidade de medicamentos que auxiliam na ereção e no desempenho sexual, à falta de informações específicas sobre a doença e até às melhores condições financeiras de alguns idosos, o que contribui para o acesso a prazeres e serviços disponíveis, permitindo uma vida sexual mais ativa 22 .

A satisfação com a vida é um julgamento pessoal pautado em alguns aspectos da vida como saúde, trabalho, condições de moradia, relações sociais, autonomia, entre outros. Alguns idosos soropositivos entrevistados em outro estudo destacaram que a falta de condições financeiras e a inexperiência com a doença também interferem negativamente na satisfação com a vida 23 .

Um estudo realizado com idosos portadores de HIV/AIDS com tempo médio de conhecimento do diagnóstico de seis anos demonstrou piores escores nas dimensões satisfação com a vida, preocupação com a saúde e aceitação do HIV em relação aos idosos entrevistados neste trabalho 24 .

Pessoas com maior tempo de diagnóstico, embora vejam o HIV/AIDS como problema, possuem elementos ligados à orientação e conformação que as beneficia. A doença passa a ser encarada como situação de aprendizagem, a cura é esperada e a adaptação considerada possível. A compreensão da doença é continuamente confrontada por diferentes diagnósticos e situações construídos pela família, amigos, vizinhos e médicos. Para o grupo com diagnóstico mais recente, as representações da AIDS estão associadas à promiscuidade, trazendo consigo o preconceito e culpabilização que acompanham esta doença 25.

A aceitação do HIV depende da percepção da condição de ser soropositivo e parece simbolizar uma oportunidade para reflexão da vida em relação ao outro e a si mesmo 26 .

Maior grau de escolaridade e melhor renda têm sido associados a escores de qualidade de vida e longevidade mais favoráveis 27 . A saúde de cada pessoa é afetada pelo comportamento individual que está associado ao status social ou econômico que tem como suporte a renda, a educação e o estado civil 28.

As pessoas com HIV ainda enfrentam alta taxa de desemprego e níveis de pobreza e, como a doença afeta principalmente pessoas em idade economicamente produtiva, o desemprego nesta população continua a ser um grande problema 29 . 
Tem sido demonstrado que pessoas com HIV/AIDS que possuem alta renda têm maior capacidade de enfrentamento da doença 30 . Além disso, outra pesquisa verificou que o rendimento anual estava associado com níveis mais baixos de percepção do estigma relacionado ao HIV. Afro-americanos, HIV positivos, com maior nível socioeconômico tiveram experiências de menor estigma relacionado ao HIV quando comparados aos afro-americanos com HIV positivos e escolaridade até o ensino médio ou com menos de 5 mil dólares de renda anual 31 .

O nível educacional interfere diretamente no desenvolvimento da consciência sanitária, na capacidade de entendimento do tratamento prescrito e na prática do autocuidado ${ }^{32}$. Existe associação estatisticamente significante entre escolaridade e conhecimento sobre a AIDS em outro estudo 33. Ainda, outros resultados sugerem que a escolaridade influencia na renda mensal, pois a escolaridade é um fator que contribui para a manutenção da atividade econômica, uma vez que pode compensar a perda da capacidade física laborativa 32 .

Os achados deste trabalho relacionados às características sociodemográficas e econômicas dos entrevistados foram semelhantes aos de outro estudo com pacientes a partir de 50 anos, em que o perfil aponta para homens, brancos, solteiros ou divorciados, aposentados, com baixo nível escolar e baixa renda 34 .

As características clínicas, epidemiológicas e comorbidades dos pacientes encontradas nesta pesquisa estão alinhadas com as de outros estudos: tempo de diagnóstico tardio 35,36, via sexual como forma mais prevalente de contaminação 34 , predomínio da contagem de linfócitos $\mathrm{T}$ CD4+ na faixa $\geq 350$ células $/ \mathrm{mm}^{3}$ no mês da entrevista 18 e doenças cardiovasculares como mais frequentes 37 .

\section{Conclusões}

Pessoas com faixa etária mais elevada começam a aparecer no cenário dos que vivem com HIV e AIDS e, diante das condições sociodemográficas e econômicas desfavoráveis em que, grande parte se encontra, este fato deve ser visto como um grande problema de saúde pública a ser enfrentado. Adicionalmente, encontramos no estudo a situação em que os idosos que vivem com HIV/ AIDS apresentaram menores escores nos domínios preocupação com o sigilo, atividade sexual e preocupação financeira. Idade, renda e tempo de diagnóstico foram as variáveis que mais responderam pela alteração da qualidade de vida.

Embora não possa ser possível generalizar os resultados deste estudo para outras populações e regiões do país, eles fornecem uma visão sobre a qualidade de vida, a relação entre qualidade de vida, aspectos socioeconômicos e clínicos de idosos com HIV/AIDS. Além disso, podem oferecer informações úteis para subsidiar as políticas de saúde na prevenção, no tratamento do HIV/ AIDS e nas intervenções de assistência social para as necessidades dos diferentes segmentos de pessoas que vivem com a doença. 


\section{Resumen}

El objetivo fue evaluar calidad de vida de adultos mayores con VIH/SIDA y correlacionarlos con sus características sociodemográficas, económicas y clínicas. Se trata de un estudio transversal analítico que evaluó a pacientes ancianos, que viven con VIH/SIDA. Se analizan factores sociodemográficos, económicos y morbilidad, de acuerdo con el Criterio de Clasificación Económica de Brasil y el Targeted Quality of Life Instrument (HAT-QoL) para evaluar la calidad de vida. Los participantes fueron 201 hombres de edad avanzada (63,7\%), solteros o divorciados (51,3\%), con perfil económico clase C, D o E (61,7\%) y baja escolaridad (53,7\%). La forma predominante de transmisión fue sexual 130 $(64,7 \%)$ y la comorbilidad más común cardiovascular 69 (34,3\%). Las variables que se correlacionaron significativamente con siete dominios de HAT-QOL fueron: edad, renta personal y familiar y tiempo transcurrido desde el diagnóstico. La calidad de vida de los pacientes se ha visto comprometida en algunos aspectos: preocupaciones sobre confidencialidad, actividad sexual y preocupación financiera. Edad, ingresos y tiempo transcurrido desde el diagnóstico fueron variables que explicaron un cambio de calidad de vida.

Anciano; VIH; Calidad de Vida

\section{Referências}

1. Programa Conjunto das Nações Unidas sobre HIV/ AIDS. Mídia - clipping: relatório global do UNAIDS 2012. http://www.unaids.org.br/documentos/clip ping.pdf (acessado em 29/Mar/2013).

2. Pereira GS, Borges CI. Conhecimento sobre HIV/ AIDS de participantes de um grupo de idosos, em Anápolis-Goiás. Esc Anna Nery Rev Enferm 2010; 14:720-5.

3. Adekeye OA, Heiman HJ, Onyeabor OS, Hyacinth HI. The new invincibles: HIV screening among older adults in the U.S. PLoS One 2012; 7:e43618.

4. Suhrcke M, Nugent RA, Stuckler D, Rocco L. Chronic disease: an economic perspective. London: Oxford Health Alliance; 2006.

5. Kirk JB, Goetz BM. Human immunodeficiency virus in an aging population, a complication of success. J Am Geriatr Soc 2009; 57:2129-38.

6. Moskowitz J, Hult J, Bussolari C, Acree M. What works in coping with HIV? A meta-analysis with implications for coping with serious illness. Psychol Bull 2009; 135:121-41.

7. Berg C, Michaelson S, Safren S. Behavioral aspects of HIV care: adherence, depression, substance use, and HIV transmission behaviors. Infect Dis Clin North Am 2007; 21:191-200.

\section{Colaboradores}

M. F. P. Okuno e A. G. S. Belasco participaram da concepção do projeto, análise e interpretação dos dados e redação do artigo; também revisaram criticamente o conteúdo intelectual do artigo e aprovaram a versão final a ser publicada. A. C. Gomes, L. Meazzini, G. Scherrer Júnior e D. Belasco Junior colaboraram na concepção, análise dos dados e redação do artigo.
8. Gore-Felton C, Koopman C. Behavioral mediation of the relationship between psychosocial factors and HIV disease progression. Psychosom Med 2008; 70:569-74.

9. Garrafa V, Godoi AMM, Soares SP. HIV/AIDS and the principle of non-discrimination and non-stigmatization. Rev Latinoam Bioét 2012; 12:118-23.

10. Cooperman NA, Arnsten JH, Klein RS. Current sexual activity and risky sexual behavior in older men with or at risk for HIV infection. Aids Educ Prev 2007; 19:321-33.

11. Kemppainen J, Bormann JE, Shively M, Kelly A, Becker S, Bone P, et al. Living with HIV: responses to a mantram intervention using the critical incident research method. J Altern Complement Med 2012; 18:76-82.

12. Tavares FMB. Apontamentos sobre o conceito de qualidade de vida: revisões, cruzamentos e possibilidades críticas. Revista Brasileira de Qualidade de Vida 2011; 3:23-32.

13. Zelaya CE, Sivaram S, Johnson SC, Srikrishnan AK, Suniti S, Celentano DD. Measurement of self, experienced, and perceived HIV/AIDS stigma using parallel scales in Chennai, India. AIDS Care 2012; 24:846-55 
14. Li L, Lee SJ, Thammawijaya P, Jiraphongsa C, Rotheram-Borus MJ. Stigma, social support, and depression among people living with HIV in Thailand. AIDS Care 2009; 21:1007-13.

15. Blanco JR, Caro AM, Pérez-Cachafeiro S, Gutiérrez F, Iribarren JA, González-García J, et al. HIV infection and aging. AIDS Rev 2010; 12:218-30.

16. Joint United Nations Programme on HIV/AIDS. Report on the global HIV/AIDS epidemic: June 1998. http://www.unaids.org/en/media/unaids/conten tassets/dataimport/pub/report/1998/19981125 global_epidemic_report_en.pdf (acessado em 29/ Mar/2013)

17. de Soárez PC, Castelo A, Abrão P, Holmes WC, Ciconelli RM. Tradução e validação de um questionário de avaliação da qualidade de vida em AIDS no Brasil. Rev Panam Salud Pública 2009; 25:69-76.

18. Lopes PSD, Silva MMG, Torres IC, Stadñik CMB. Qualidade de vida dos pacientes HIV positivo com mais de 50 anos. Rev AMRIGS 2011; 55:356-60.

19. Joint United Nations Programme on HIV/AIDS. Reducing HIV stigma and discrimination: a critical part of national AIDS programmes. http://data. unaids.org/pub/Report/2008/JC1521_stigmatisa tion_en.pdf (acessado em 29/Mar/2013).

20. Silva J, Saldanha AAW, Azevedo RLW. Variáveis de impacto na qualidade de vida de pessoas acima de 50 anos HIV+. Psicol Reflex Crit 2010; 23:56-63.

21. Andrade HAS, Silva SK, Santos MIPO. AIDS em idosos: vivências dos doentes. Esc Anna Nery Rev Enferm 2010; 14:712-9.

22. Bertoncini BZ, Moraes KS, Kulkamp IC. Comportamento sexual em adultos maiores de 50 anos infectados pelo HIV. DST J Bras Doenças Sex Transm 2007; 19:75-9

23. Tahan J, Carvalho ACD. Reflexões de idosos participantes de grupos de promoção de saúde acerca do envelhecimento e da qualidade de vida. Saúde Soc 2010; 19:878-88

24. Gil NLM, Souza LR. Qualidade de vida de indivíduos infectados pelo HIV relacionada com as características sociodemográficas e clínicas. Ciênc Cuid Saúde 2010; 9:697-703.

25. Saldanha AAW, Araújo LF, Sousa VC. Envelhecer com AIDS: representações, crenças e atitudes de idosos soropositivos para o HIV. Interam J Psychol 2009; 43:323-32.

26. Teixeira MG, Silva GA. A representação do portador do vírus da imunodeficiência humana sobre o tratamento com os anti-retrovirais. Rev Esc Enferm USP 2008; 42:729-36.
27. Santos SAL, Tavares DMS, Barbosa MH. Fatores socioeconômicos, incapacidade funcional e número de doenças entre idosos. Rev Eletrônica Enferm 2010; 12:692-7.

28. Song R, Hall HI, Harrison KM, Sharpe TT, Lin LS, Dean HD. Identifying the impact of social determinants of health on disease rates using correlation analysis of area-based summary information. Public Health Rep 2011; 126 Suppl 3:70-80.

29. Oliva J. Labour participation of people living with HIV/AIDS in Spain. Health Econ 2010; 19:491-500.

30. Rajeev KH, Yuvaraj BY, Nagendra Gowda MR, Ravikumar SM. Impact of HIV/AIDS on quality of life of people living with HIV/AIDS in Chitradurga district, Karnataka. Indian J Public Health 2012; 56:116-21.

31. Galvan FH, Davis EM, Banks D, Bing EG. HIV stigma and social support among African Americans. AIDS Patient Care STDS 2008; 22:423-36.

32. Reis LA, Torres GV, Reis LA. Características sóciodemográficas e de saúde de idosos de uma instituição do Município de Jequié/BA. Espaç Saúde 2008; 9:39-46.

33. Batista AFO, Marques APO, Leal MCC, Maino JE, Melo HMA. Idosos: associação entre o conhecimento da aids, atividade sexual e condições sociodemográficas. Rev Bras Geriatr Gerontol 2011; 14:39-48

34. Ultramari L, Moretto PB, Gir E, Canini SRMS, Teles SA, Gaspar J, et al. Perfil clínico e epidemiológico da infecção pelo HIV/aids em idosos. Rev Eletrônica Enferm 2011; 13:405-12.

35. Brookmeyer R. Reconstruction and future trends of the AIDS epidemic in the United States. Science 1991; 253:37-42.

36. Fernandes JRM, Acurcio FA, Campos LN, Guimarães MDC. Início da terapia anti-retroviral em estágio avançado de imunodeficiência entre indivíduos portadores de HIV/AIDS em Belo Horizonte, Minas Gerais, Brasil. Cad Saúde Pública 2009; 25:1369-80

37. Barbaro G, Barbarini G. Human immunodeficiency virus \& cardiovascular risk. Indian J Med Res 2011; 134:898-903.

Recebido em 07/Mai/2013

Versão final reapresentada em 09/Jan/2014

Aprovado em 15/Jan/2014 